\title{
Discovery of large-scale masers in W3(OH)
}

\author{
L. Harvey-Smith ${ }^{1}$ and R. J. Cohen ${ }^{2}$ \\ ${ }^{1}$ Joint Institute for VLBI in Europe, Postbus 2, 7990 AA, The Netherlands. harvey@jive.nl \\ ${ }^{2}$ Jodrell Bank Observatory, Macclesfield, Cheshire, SK11 9DL, UK. rjc@jb.man.ac.uk
}

\begin{abstract}
We report a vast filament of hydroxyl and methanol maser emission surrounding the ultra-compact $\mathrm{HII}$ region $\mathrm{W} 3(\mathrm{OH})$. The filament stretches $3100 \mathrm{AU}$ and has a linear velocity gradient. By studying the velocity structure, line profiles and extended methanol maser structures we believe we have located the position of the central star and detected around it a circumstellar disc with a large velocity gradient of $47 \mathrm{~km} \mathrm{~s}^{-1} \operatorname{arcsec}^{-1}$.
\end{abstract}

Keywords. masers, (ISM:) HII regions, ISM: molecules, radio lines: ISM, stars: formation

\section{Introduction}

Despite playing a vital and dominant role in feedback processes in the interstellar medium, massive stars are still not understood in terms of their formation processes. Hydroxyl and methanol masers are bright tracers of the early stages of massive starformation, allowing us to study these objects at milliarcsecond resolution.

\section{Discovery of an extended maser filament in $\mathrm{W} 3(\mathrm{OH})$}

Using MERLIN we observed the $\mathrm{OH}$ and methanol masers around the ucHII region $\mathrm{W} 3(\mathrm{OH})$. For the first time, we recovered all the single-dish flux in both species. This allowed us to discover a vast (3100 AU) filament of masing gas stretching north-south across the western face of W3(OH) (Harvey-Smith \& Cohen 2005). The filament has a linear velocity gradient along its length, which is seen in both methanol and hydroxyl molecules (Harvey-Smith \& Cohen 2006). The extended methanol and $\mathrm{OH}$ masers tend to avoid each other on small ( 0.1 arcsecond) scales, but together trace a continuous path across the face of the ucHiI region.

\section{A possible circumstellar disc in $\mathrm{W} 3(\mathrm{OH})$}

Within the extended maser filament we find a small region with distinct physical properties. This region contains the brightest $6.7-\mathrm{GHz}$ and $12.2-\mathrm{GHz}$ methanol masers, the 13.4-GHz OH maser peak, the brightest radio continuum emission and the strongest magnetic field in $\mathrm{W} 3(\mathrm{OH})$. At this location we have discovered a small 6.7-GHz methanol maser filament with broad line profiles and a linear velocity gradient of $4990 \mathrm{~km} \mathrm{~s}^{-1} \mathrm{pc}^{-1}$. This is consistent with an edge-on circumstellar disc around a central star with a mass $>13 \mathrm{M}_{\odot}$. We suggest that this broadline feature houses the central star or stars powering the ultra-compact HiI region.

\section{References}

Harvey-Smith, L. \& Cohen, R.J. 2005, MNRAS 356, 637

Harvey-Smith, L. \& Cohen, R.J. 2006, MNRAS 371, 1550 